\title{
Germline variants in SMARCB1 and other members of the BAF chromatin-remodeling complex across human disease entities: a meta-analysis
}

\author{
Till Holsten ${ }^{1,2,3} \cdot$ Susanne Bens ${ }^{4} \cdot$ Florian Oyen $^{1} \cdot$ Karolina Nemes $^{5} \cdot$ Martin Hasselblatt $^{6} \cdot$ Uwe Kordes $^{1}$. \\ Reiner Siebert ${ }^{4} \cdot$ Michael C. Frühwald ${ }^{5} \cdot$ Reinhard Schneppenheim ${ }^{1} \cdot$ Ulrich Schüller $\left(\mathbb{D}^{1,2,3}\right.$
}

Received: 15 May 2017 / Revised: 27 February 2018 / Accepted: 13 March 2018 / Published online: 30 April 2018

(c) European Society of Human Genetics 2018

\begin{abstract}
Germline variants that affect function are found in seven genes of the BAF chromatin-remodeling complex. They are linked to a broad range of diseases that, according to the gene affected, range from non-syndromic or syndromic neurodevelopmental disorders to low-grade tumors and malignancies. In the current meta-analysis, we evaluate genetic and clinical data from more than 400 families and 577 patients affected by $B A F$ germline alterations. We focus on SMARCB1, including 43 unpublished patients from the EU-RHAB registry and our institution. For this gene, we further demonstrate whole gene as well as exon deletions and truncating variants to be associated with malignancy and early-onset disease. In contrast, non-truncating variants are associated with non-malignant disorders, such as Coffin-Siris syndrome or late-onset tumors like schwannoma or meningioma $(p<0.0001)$. SMARCB1 germline variants are distributed across the gene with variants in exons $1,2,8$, and 9 being associated with low-grade entities, and single-nucleotide variants or indels outside of exon 9 that appear in patients with malignancies $(p<0.001)$. We attribute variants in specific $B A F$ genes to certain disease entities. Finally, single-nucleotide variants and indels are sometimes detected in the healthy relatives of tumor patients, while Coffin-Siris syndrome and Nicolaides-Baraitser syndrome generally seem to appear de novo. Our findings add further information on the genotype-phenotype association of germline variants detected in genes of the BAF complex. Functional studies are urgently needed for a deeper understanding of $B A F$-related disorders and may take advantage from the comprehensive information gathered in this article.
\end{abstract}

\section{Introduction}

Germline variants in subunits of the BAF (BRG1/BRMassociated factor) or SWItch/sucrose non-fermentable (SWI/SNF) chromatin-remodeling complex have recently been implicated in benign and malignant tumors as well as neurodevelopmental disorders, always depending on the gene affected. $S W I / S N F$ is highly conserved from yeast to

These authors contributed equally: Reinhard Schneppenheim, Ulrich Schüller

Electronic supplementary material The online version of this article (https://doi.org/10.1038/s41431-018-0143-1) contains supplementary material, which is available to authorized users.

Ulrich Schüller

u.schueller@uke.de

Extended author information available on the last page of the article its human analog $B A F$, but also displays certain differences in composition and function. These genes encode for a group of proteins that associate to alter the spatial configuration of the DNA in an ATP-dependent manner and hereby regulate its accessibility for transcription factors. BAF functions as an epigenetic modulator of cell differentiation [1], metabolism [2], fate [3], DNA repair [4], and as a tumor suppressor [5]. Latest findings illuminate the role of BAF as a regulator of enhancers associated with developmental processes, an interaction that may be perturbed by loss of its SMARCB1/BAF47 subunit [6, 7]. In total, the complex is composed of 12-28 subunits and always includes the core units SMARCB1/BAF47, SMARCC1/ BAF155, and the mutually exclusive ATPase SMARCA4/ BAF190A or SMARCA2/BAF190 as a catalytic effector molecule. Mammalian BAF complexes divide into Brahmaassociated factor (BAF) and Polybromo BAF (PBAF) complexes, defined by the presence of either ARID1A/B/ BAF250A/B or ARID2/BAF200 and PBRM1/BAF180 [8]. 
Other defining subunits vary depending on cell type and tissue, enabling the diverse functions of the complex. Considering its versatile regulatory role, it is unsurprising that mutational events in $B A F$ genes disturb cellular processes ranging from early development to cell survival and death. Indeed, up to $20 \%$ of human cancers harbor somatic gene variants in $B A F$ genes, including a large variety of carcinomas, pediatric malignancies, and low-grade tumors [9]. The role of the $B A F$ subunit $S M A R C B 1$ as a tumor suppressor is best understood, as it is closely linked to rhabdoid tumors (RT) [5]. Rhabdoid tumors are rare and highly malignant pediatric cancers that may occur in the CNS (Atypical teratoid/rhabdoid tumor: AT/RT), the kidney (Rhabdoid tumor of the kidney: RTK), and soft tissue (Malignant rhabdoid tumor: MRT) predominantly of infants and young children. Their clinical heterogeneity contrasts with a remarkable simple genome, characterized by biallelic $S M A R C B 1$ inactivation as the only recurring feature. This follows a classical two-hit model with germline events in up to $30 \%$ of cases (rhabdoid tumor predisposition syndrome type 1, OMIM \# 609322) [10]. SMARCA4 variants may rarely also function in the same manner (rhabdoid tumor predisposition syndrome type 2, OMIM \# 613325) [11, 12].

Exomic sequencing of adult and pediatric cancers has discovered somatic gene variants in many units of the BAF complex. However, germline variants linked to the development of solid tumors have only been detected in SMARCA4, SMARCB1, and SMARCE1 [13]. Constitutional (mostly de novo) variants of SMARCBI and SMARCA4 as a first step followed by somatic loss-of-heterozygosity (LOH) represent a paradigmatic two-hit tumor model of epigenetic regulators. Most prominently they lead to RT, or more rarely (SMARCB1 only) to cribriform neuroepithelial tumors (CRINET) [14], malignant peripheral nerve sheath tumors (MPNST) [15, 16], epitheloid sarcomas [17], leiomyosarcomas [18], or schwannomas (SWNTS1, OMIM \# 162091) [16] (mosaic LOH or haplo-insufficiency in some cases is sufficient regarding the last three entities; in case of schwannoma changes in other genes may be required additionally). SMARCA4 germline variants may lead to small-cell carcinomas of the ovary, hypercalcaemic type (SCCOHT) [19, 20]. SMARCE1 variants are restricted to non-NF2-driven clear cell meningioma (OMIM \# 607174) [21].

Variant carriers suffering from intellectual disability or specific neurodevelopmental disorders constitute a second group of patients with currently little evidence for tumor predisposition, although follow-up data from larger groups of patients are lacking. Heterozygous variants in five genes encoding subunits of BAF account for the majority of the clinically heterogeneous Coffin-Siris syndrome (CSS, OMIM \# 135900), while the clinically more homogenous Nicolaides-Baraitser syndrome (NCBRS, OMIM \# 601358) appears to be due to SMARCA2 germline variants. Both rely on genetic variants of one copy of the respective genes. Key findings shared in both syndromes include intellectual disability and coarse facial features, distinguishing findings in CSS are thumb/toe hypoplasia and hypertrichosis vs. thickened interphalangeal joints and sparse hair in NCBRS [22]. Penetrance for both syndromes appears to be complete $[23,24]$. Interestingly, the co-occurrence of tumors in such patients is restricted to anecdotal reports [25-27].

In the current meta-analysis we link clinical and genetic data from over 400 families with 577 patients carrying $B A F$ germline alterations. We include 43 unpublished cases and discuss potential mechanisms causal for pathogenesis in order to decipher genotype-phenotype relations.

\section{Methods}

\section{Published cases with BAF germline variants}

We reviewed the literature, eventually including 534 BAF germline variant carriers from more than 60 published manuscripts and from the open source SMARCB1 database that gathers patients suffering from SMARCB1-deficient neoplasia with special regards on tumor genetics (https://www.uke.de/dateien/kliniken/paediatrische-haema tologie-und-onkologie/dokumente/smarcb1/smarcb1_data_ uke_2009-11-26.html, April 20th 2017). Pubmed-based investigation included the following search items: "BAF/ SWI/SNF germline mutation/alteration/variant", or "BAF/ SWI/SNF constitutional mutation/alteration/variant" supplemented each by the terms "SMARCA2", "SMARCA4", "SMARCB1", "SMARCC1", "SMARCE1", "ARID1A", "ARID1B", “ARID2", "BRM", "BRG1", "INI1", "BAF190", "BAF190A", "BAF155", "BAF57", "BAF250a”, BAF250b, "BAF200”, "tumor", "neoplasia”, "malignancy", "rhabdoid tumor", “ATRT”, “AT/RT”, "MRT”, "RTK”, “CRINET”, "SCCOHT”, "schwannoma”, "schwannomatosis", "meningioma", "autism", "CSS", "Coffin Siris syndrome”, "NCBRS”, "Nicolaides-Baraitser syndrome". Patients were included in our study whenever the diagnosis had been verified genetically, and information on the germline status was given.

\section{Novel cases with BAF germline variants}

Twenty-six patients included in this study are derived from the European rhabdoid registry-cohort (EU-RHAB), another 17 were gathered from our own archives. All individuals are listed in Supplementary Table 1, organized in respect to diagnosis, variant, and relationship to other variant carriers. If already published, the respective literature is cited. Supplementary Table 2 lists families with more than one known 
carrier of a $B A F$ germline variant separately, while Supplementary Table 3 gives an overview of individuals and families suffering from more than one disease. Unpublished individuals have been submitted to the LOVD-database for public insight: https://databases.lovd.nl/shared/genes/ SMARCB1 (\# 00107735-00107756 and \# 0010781100107831).

\section{Statistical analysis}

We used $\chi^{2}$ analysis to determine significance of differences between reference groups. Level of significance was set at $<0.05$. If patients suffered from more than one disease, we counted every single entity arising as one separate case: Comparing "cases of low-grade tumor" vs. "cases of malignant tumor", a patient bearing schwannoma as well as MPNST contributes to both groups. If a patient fell ill with different types of rhabdoid tumor, this was considered as one entity.

\section{Results}

\section{BAF-related diseases can be attributed to variants within specific BAF genes}

Screening by unbiased exomic sequencing in different tumor cohorts and patients with unexplained intellectual disability has revealed an ever expanding relation of various diseases to $B A F$ alterations [9]. An overview on diseases related to $B A F$ germline variants is given in Table 1 . Alterations in SMARCBI represent the most common genetic change that predisposes to the development of BAFrelated diseases (339 SMARCB1 variant carriers/577 BAF cases in total). SMARCB1 germline variant carriers also suffer from a broader range of different entities compared to other $B A F$ complex gene germline variant carriers. Constitutional variants in SMARCB1 predispose, for instance, to the rhabdoid tumor predisposition syndrome type $1(n=$ 185 RTPS1/339 SMARCB1 variant carriers), resulting in early-onset highly malignant tumors [28]. These patients are affected by rhabdoid tumors at any anatomical site including CNS $(n=109$ AT/RT/185 SMARCB1 mutated RT, including 27 unpublished cases), kidney ( $n=12$ RTK/185, including one unpublished case), and soft tissue ( $n=24$ MRT/185, including five unpublished cases). Of note, a significant proportion of patients presented with bi- and multifocal tumors $(n=39$ multifocal RT/185, including ten unpublished cases). SMARCB1 germline variants have further been detected in patients with schwannomatosis (89/ 339 SMARCB1 variant carriers) or CRINET (3/339) [14]. Except for SMARCA4, no other BAF complex member encoding gene has been shown to be involved in the

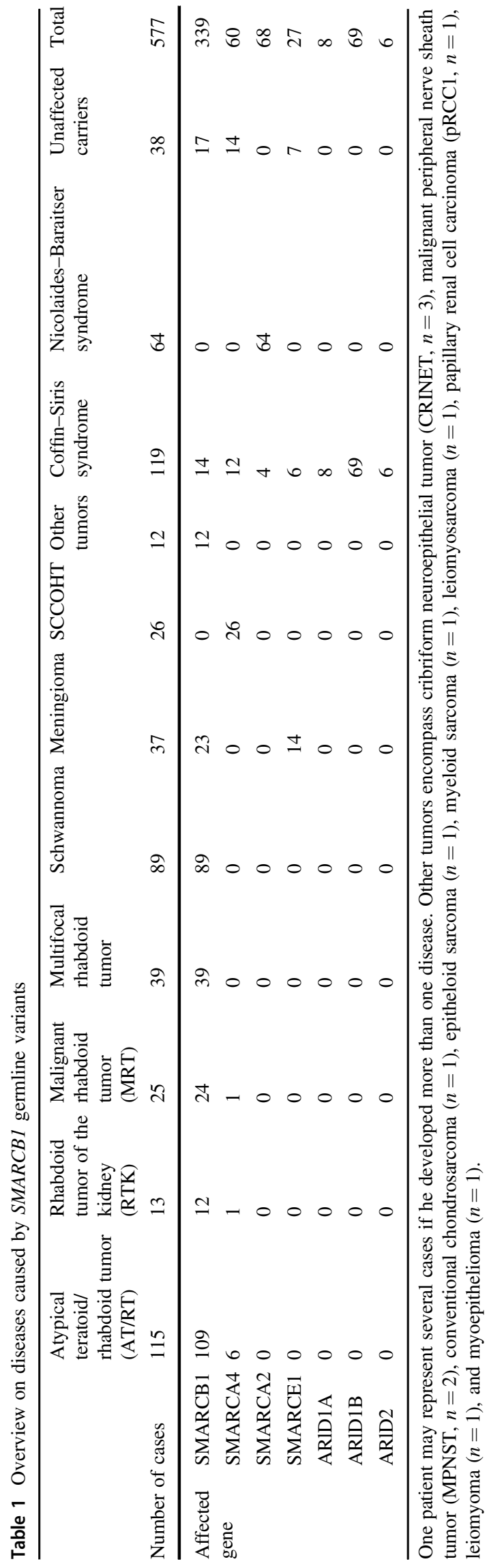


tumorigenesis of these diseases. Very rarely, other neoplasms may arise in the setting of a SMARCB1 germline variant, i.e., meningioma $(n=23 / 339)$ [21, 29, 30], malignant peripheral nerve sheath tumor $(n=2 / 339)$ [15, 16], chondrosarcoma $(n=1 / 339)$ [31], epitheloid sarcoma $(n=$ 1/339) [17], leiomyosarcoma $(n=1 / 339)$ [18], leiomyoma $(n=1 / 339)$ [32], and myoepithelioma $(n=1 / 339)$ [33]. In addition to neoplastic diseases, there are a few cases of patients with CSS, who carry SMARCB1 germline variants $(n=14 / 339)[22,34,35]$.

The disease spectrum of patients with SMARCA4 variants partially resembles the SMARCB1 spectrum of diseases as they are known to cause the RTPS2 $(n=8 / 60$ SMARCA4 variant carriers) $[10,11]$ and, likewise, may be detected in patients with Coffin-Siris syndrome $(n=12 / 60)$ $[34,35]$. However, SMARCA4 is the only $B A F$ gene that has been mentioned in the context of small cell carcinoma of the ovary hypercalcaemic type (SCCOHT) involving both somatic and germline variants $(n=26 / 60)$ [20]. So far, 14 unaffected variant carriers $(n=14 / 60)$ have been published, indicating incomplete penetrance (Table 3 and Supplementary Table 1).

SMARCA2 germline variants $(n=68)$ have not been implicated in tumorigenesis, but they are the only known gene linked to NCBRS [36]. The small number of patients with CSS attributed to SMARCA2 variants $(n=4)$ have been reclassified as suffering from NCBRS [35].

Constitutional SMARCE1 variants are less pleiotropic and are seen in association with predominantly spinal clear cell meningeoma ( $n=14 / 27$ SMARCE1 variant carriers) [21]. Few Coffin-Siris patients $(n=6 / 27)$ [22, 25, 34, 35] as well as healthy SMARCE1 variant carriers in families with multiple relatives affected by meningiomas $(n=7 / 27)$ [37-39] have also been published.

Germline alterations of ARIDI/2 genes (ARIDIA, $A R I D 1 B, A R I D 2)$ have only been linked to CoffinSiris syndrome with ARIDIB variants (69/83 ARIDI/2 variant carriers) as the most common genetic change for this neurodevelopmental disorder so far [22, 34, 35]. Intriguingly, truncating ARIDI/2 variants seem to have different impact on the phenotype depending on whether they occur in the germline, causing CSS, or as somatic events with an association to tumor development [40-42]. The preponderance of $A R I D I B$ variations could be explained by the hypothesis that full ARIDIA deletions are lethal, a fact that is underlined by the circumstance that most ARIDIA variations in CSS patients occur as a mosaic $[22,35]$.

Surpassing all $B A F$ genes, SMARCBI germline variants have the highest pleiotropy. This includes malignant as well as low-grade tumors and neurodevelopmental disorders, most prominently CSS, but also individuals with EHMTInegative Kleefstra syndrome [43]. In contrast, variants in
SMARCA4, SMARCA2, SMARCE1, ARIDIA, ARID1B, and $A R I D 2$ each are clinically more distinct.

\section{Type of SMARCB1 variant determines type of disease}

We next took a closer look on the type of variants occurring within SMARCB1, correlating them with resulting phenotypes (Table 2). We focussed on SMARCBI as the most pleiotropic gene of the $B A F$ family members.

Truncating SMARCBI variants (whole gene deletions, partial deletions, nonsense, and frameshift variants) are much more likely to be associated with malignancies $(p<$ $0.001, \chi^{2}$ analysis). While partial and whole gene deletions of SMARCBI only appear in the setting of rhabdoid tumors, this entity rarely arises due to SMARCBI missense or inframe variants. We identified one single patient with a SMARCB1 missense variant (c.1142 C > G p.Thr381Arg) suffering from a CRINET [14]. Instead, SMARCB1 missense variants display a clear association with low-grade tumor entities and non-oncologic illnesses (benign: $n=34 /$ 42, malignant: $n=1 / 42$, non-oncologic: $n=4 / 42$, unaffected carriers 3/42). Indeed, in all 13 Coffin-Siris SMARCBI variant carriers in our study an in-frame or missense variant provides the genetic basis for the disease (Supplementary Table 1). These variants cluster closely at exon 8 and 9, indicating for a specific position effect in the pathogenesis of SMARCB1-related CSS. Development of schwannoma and meningioma is more complex and involves other co-operating genes such as NF2 and LZTRI [21, 44]. SMARCB1-Schwannomatosis (SWNTS1) is characterized by spinal and often painful peripheral schwannomas excluding acoustic neurinoma, that appear in the setting of SMARCB1 nonsense- and frameshift variants, but not exon-spanning deletions, which carry a high risk for malignancy. Splice-site variants are detectable in several tumor entities of both benign and malignant clinical behavior.

The most frequent combination of entities arising simultaneously is schwannoma plus meningioma. So far four families and two further patients have been published. The families include 16 patients, who developed both schwannoma and meningioma, while four individuals exclusively suffered from meningioma, four others from schwannoma, and three healthy carriers were observed (Supplementary Table 2).

SWNTS1 may also encompass RT, however. Of note, one patient additionally developed an MPNST within a schwannoma (Family 5: AT/RT $n=2$, schwannoma $n=3$, no evidence of disease (NEoD) $n=1$ [45]; Family 6: AT/ RT $n=2$, schwannoma + MPNST $n=1$, NEoD $n=1$ [15]), and there is one further report of a SMARCB1 germline variant carrier with schwannoma and MPNST 


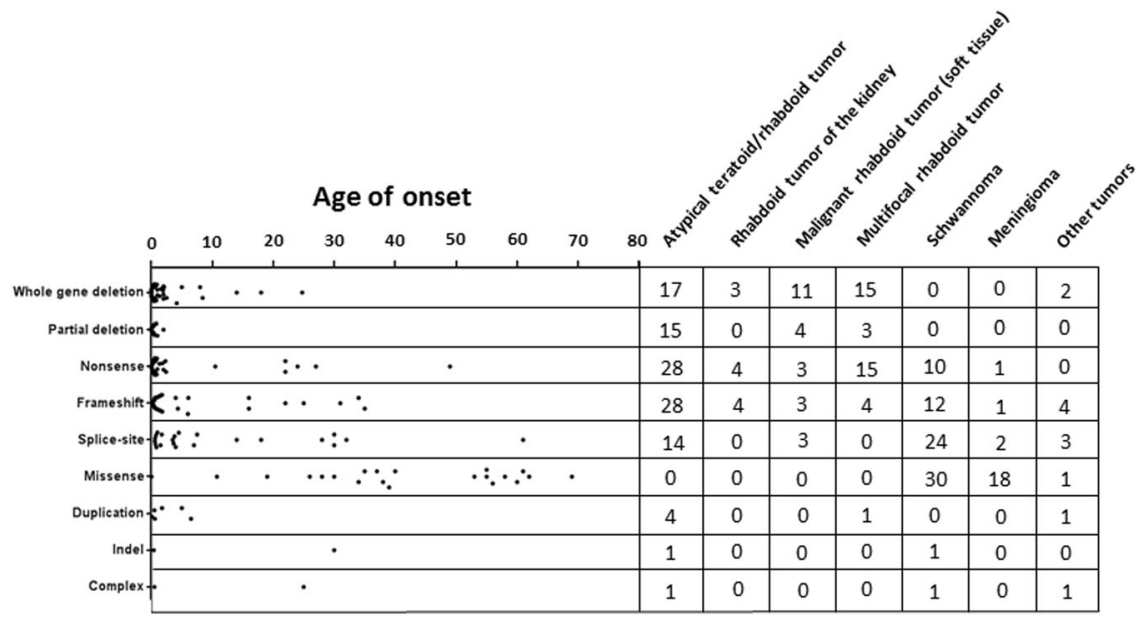

Dots indicate single patients. Numbers count for cases of disease, so that patients with more than one disease may contribute to more than one category. Other tumors encompass cribriform neuroepithelial tumor (CRINET, $n=3$ ), malignant peripheral nerve sheath tumor (MPNST, $n=2$ ), myeloid sarcoma $(n=1)$, epitheloid sarcoma $(n=1)$, conventional chondrosarcoma $(n=1)$, leiomyosarcoma $(n=1)$, leiomyoma $(n=1)$, papillary renal cell carcinoma type $1(\mathrm{pRCC} 1, n=1)$ and myoepithelioma $(n=1)$.

[16]. A SMARCB1-negative leiomyoma as well as a leiomyosarcoma both affected schwannoma patients [18, 32]. Furthermore, Gossai et al. published a Coffin-Siris patient, diagnosed with schwannomatosis-induced cord compression at 33 years of age [25]. Another interesting case is a former MRT patient reported by Forest et al., who subsequently developed chondrosarcoma [31]. A further family of six individuals had two healthy variant carriers, while three members were diagnosed with AT/RT in childhood. Of the latter, one developed a SMARCB1-negative myoepithelioma later in life. The sixth member died at age of 0.6 years from a posterior fossa mass not further analyzed [33].

Categorizing variant carriers with regard to their specific germline event reveals clear differences, but also some overlaps. Exon-spanning deletions are solely linked to the generation of RT, whereas non-truncating variants only rarely predispose to malignancies. Nonsense, frameshift, and splice-site variants exhibit varying effects concerning development of malignant or non-malignant disease.

\section{SMARCB1 variant determines age of disease onset}

In order to evaluate genetic and clinical variables, we plotted age of disease onset and type of variant of 160 SMARCB1 germline variant carriers with tumor disease. We found that the distribution across time depends on the type of the underlying variant: Truncating variants are associated with early-onset disease, non-truncating variants with lateonset disease (average age 45 months vs. 519.5 months, $p<$ 0.0001 ; median age of onset 7 months vs. 474 months, Table 2). Missense variants in SMARCBI are detected in individuals not diagnosed with tumors before their 10th year of age. Disregarding two exceptional patients with constitutional whole gene deletion and diagnosis of RT at age 14 and 24.5 years $[10,46]$, there is a strong correlation between functional impact of a SMARCB1 germline variant and age of disease onset. This rule applies also to low-grade SMARCBI tumors with truncating (median age at onset: 294 months) vs. non-truncating germline variants (median age at onset: 474 months, average age 308 vs. 519 months, $p<0.0005)$.

\section{SMARCB1-associated tumor entities show characteristic variation patterns}

To gain a detailed overview of the genomic landscape of SMARCBI germline events we arranged the variants along the gene, sorting them by malignant or benign clinical behavior (Fig. 1). While single-nucleotide variants associated with malignancies tend to be located in the center of the gene $(61 / 98$, on exons $3-7 ; 37 / 98,38 \%$ on exons $1,2,8,9$ ), such that appear in the context of schwannoma, meningioma, or Coffin-Siris syndrome are predominantly found on its ends $(52 / 63,82.5 \%$ on exons 1 , $2,8,9 ; 11 / 63,17.5 \%$ on exons $3-7 ; p<0.001)$. Strikingly, variants in the MYC binding, the HIV integrase binding, and the PPP1R15A interacting domain are exclusively associated with development of rhabdoid tumors. While variants in the DNA binding site or the SNF5 domain may also predispose to schwannoma or meningioma, those leading to CRINET or CSS reside outside specific domains known so far. 
SMARCB1

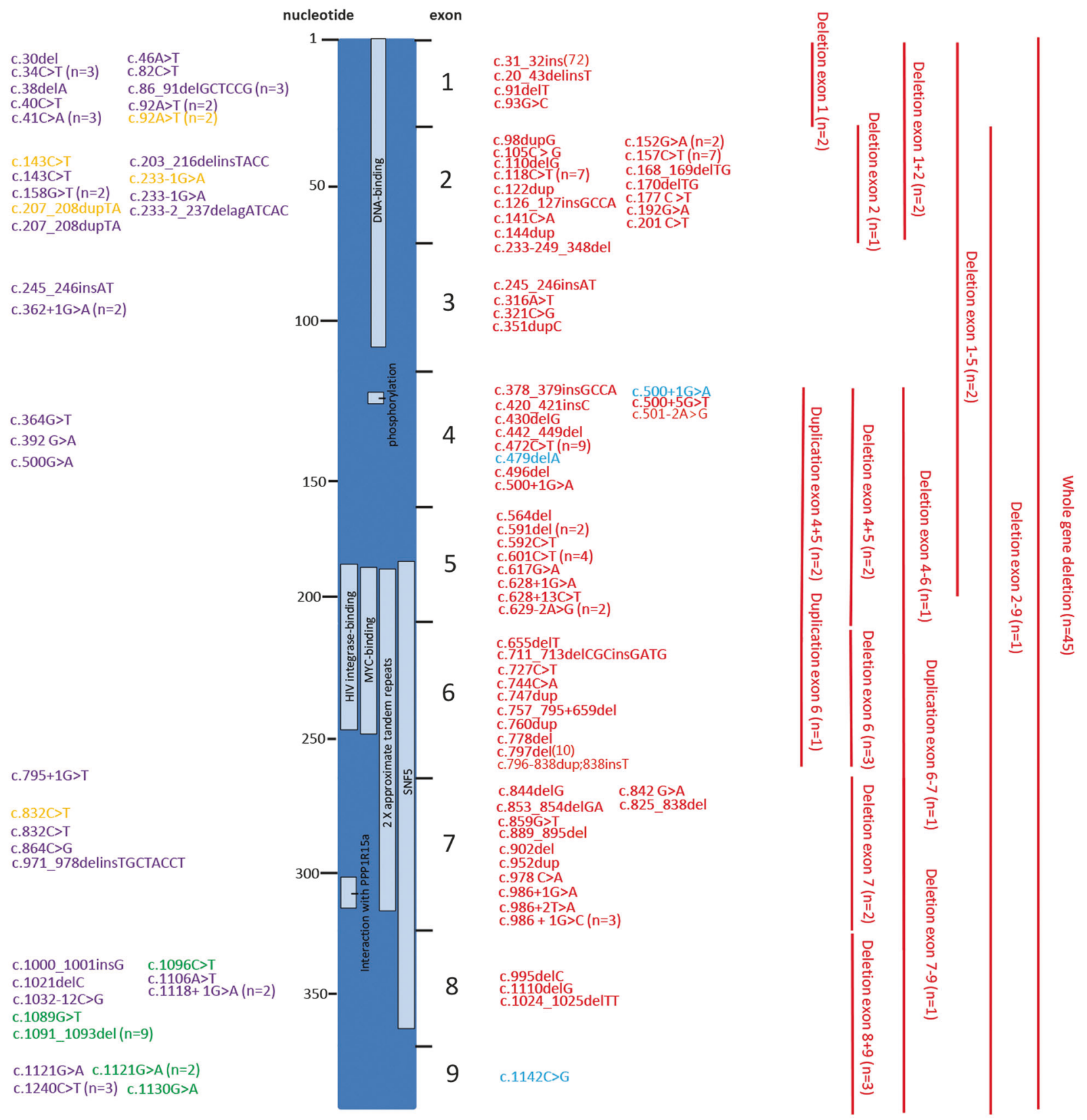

\begin{tabular}{|l|l|}
\hline Schwannoma & CRINET \\
Meningioma & Rhabdoid tumor \\
Coffin-Siris syndrome & \\
\hline
\end{tabular}

Fig. 1 Distribution and type of germline variants occuring along the SMARCB1 gene. On the left side, variants causing benign entities are indicated in violet (schwannoma), yellow (meningioma), and green (Coffin-Siris syndrome), while the right side shows malignancies in red (rhabdoid tumor) and light blue (CRINET). Red bars show extent of deletions and duplications. Note that variants in benign disorders are mainly present in $1,2,8$, and 9 , while malignant tumors are frequently caused by variants within exons $2-7$. Light blue bars indicate for known domains 
Table 3 Distribution of BAF germline alterations in families with unaffected variant carriers

\begin{tabular}{|c|c|c|c|c|c|c|c|c|c|}
\hline $\begin{array}{l}\text { Whole gene } \\
\text { deletion }\end{array}$ & $\begin{array}{l}\text { Partial } \\
\text { deletion }\end{array}$ & Nonsense & Missense & Frameshift & In-frame & Splice-site & Duplication & Indel & $\begin{array}{l}\text { Total number } \\
\text { of families }\end{array}$ \\
\hline 0 & 2 & 1 & 1 & 3 & 0 & 4 & 1 & 0 & 12 \\
\hline 0 & 0 & 3 & 1 & 1 & 0 & 0 & 0 & 0 & 5 \\
\hline 0 & 1 & 0 & 0 & 2 & 0 & 0 & 0 & 1 & 4 \\
\hline
\end{tabular}

Healthy individuals with germline mutations in SMARCA2, ARIDIA, ARIDIB, and ARID2 are not known.

\section{Unaffected BAF germline variant carriers harbor diverse types of variants excluding whole gene deletions and in-frame variants}

Table 3 summarizes family screening results depicting the prevalence of healthy carriers related to index tumor patients with constitutional BAF-related disease. This involves individuals with variants in SMARCB1 (17/38 unaffected variant carriers in total) $[10,14,15,29,33,45$, 47-51], SMARCA4 (14/38) [11, 12, 52, 53], and SMARCE1 (7/38) [37-39], but no healthy carriers of $A R I D I A / B$ or $A R I D 2$ variants $(0 / 38)$. Referred to all variant carriers of a particular gene, the incidence of unaffected individuals is by far higher among SMARCE1 and SMARCA4 variant carriers $(7 / 27$ and 14/60) than in carriers of SMARCBI variants (17/339), suggesting incomplete penetrance for variant carriers with SMARCB1 variants, that is even further reduced regarding the SMARCA4 and SMARCE1 situation. Truncating as well as non-truncating variants were found. Of interest, neither whole gene deletions nor inframe variants appear in this context (0/38). The largest group is represented by splice-site variants in SMARCBI $(6 / 38)$.

\section{Discussion}

Reviewing and extending the number of $B A F$ germline variant carriers known so far provides insight into a wide landscape of various genetic events with diverging impact on a carrier's phenotype.

Considering the phenotypic range from aggressive tumors to asymptomatic individuals, the intriguing question concerns the underlying modifying effectors. Though up to $20 \%$ of human cancers harbor $B A F$ variants [9], research focussing exclusively on germline variants usually is restricted to very small cohorts of patients. Thus, metaanalyses are required to gain an overview.

Hence, the scope of our work was to broaden the perspective on $B A F$ genetics by integrating new and existing data with regard to disease spectrum, possible pathomechanisms, and clinical data. Affecting a core $B A F$ member, germline alterations in SMARCB1 have first been related to the development of rhabdoid tumors in the late nineties [54]. While this finding has been confirmed in numerous studies, further research suggested other entities to be involved as well. Among others, schwannoma, meningioma, and CSS were ascertained to arise in the setting of SMARCB1 germline variants. Detailed consideration of the specific genetic events reveals that SMARCB1-related rhabdoid tumors are exclusively associated with truncating variants. It is noteworthy that variants occuring in more benign tumor entities or CSS tend to affect the first and last exons, whereas such causing RT either represent as exonspanning or complete SMARCBI deletions or singlenucleotide variants and indels that widely spare out exon 1. For exon 9, it is known that variants do not lead to nonsense-mediated decay, which might explain the apparent lack of changes associated with malignancy here. Variants within the center of the gene (MYC binding, HIV integrase binding, and interaction with PPP1R15A) may predispose to rhabdoid tumors, while those at the beginning of SMARCBI (DNA binding) may additionally lead to schwannoma or meningioma. A series of variants cannot be directly linked to any known domain, including all singlenucleotide variants resulting in CRINET and CSS. Future 3D modeling of the protein structure and its interfaces with other proteins of the complex along with functional analyses might help to understand this peculiar pattern. However, investigating the functional impact of selected variants may be of high value for a deeper understanding of $B A F$ genetics.

Our data strengthens the results Smith et al. demonstrated in a smaller study regarding schwannoma and RT. The overview (Fig. 1) points out that this phenomenon may also hold true for other diseases, emphasizing the role of the first and last exons of SMARCB1 especially in the generation of non-malignant disease [16]. Nevertheless, a few truncating variants in exon 1,2 , and 8 , predispose to malignant tumors. Presuming a consistent frequency of variation along the gene, these observations might indicate a particular susceptibility for its first and last exons, as nontruncating variants in its center may indeed exist, but obviously do not become apparent in a clinical setting. To date, deletions of every SMARCB1 exon have been detected. Independent of their extent (ranging from one exon up to whole gene deletion) they categorically lead to formation of RT. 
The effectors modifying the very discordant outcome of variations probably affecting function in the BAF complex remain largely unknown (It is obvious that severity of a disease depends on the functional alterations of the protein a mutated gene encodes; still it remains unclear, why the same type of variant or, in rare cases, the very same variant might be related to both, a benign tumor and a malignancy.). In our cohort, three types of variants-nonsense, frameshift, and splice-site variants-predispose to RT and schwannoma as well. Santen et al. further state that germline variants in $A R I D I A / B$ and SMARCBI causing mental retardation syndromes can also be found as somatic variants in tumor patients [55]. Moreover, one patient with both CSS and schwannoma and a SMARCB1 germline variant has been reported [25]. The combination of intellectual disability and malignancy due to germline alterations interestingly seems uncommon. While the impact of splice-site variants generally is hard to predict, nonsense and frameshift variants can result in protein truncation. However, the different outcome of affected individuals suggests that in some cases there might be residual protein activity, potentially in form of a variant leaving at least parts of the protein functional. This assumption is supported by data derived from Hulsebos et al., who expressed four schwannomatosisassociated SMARCBI exon 1 variants in rhabdoid tumor cells lacking endogenous SMARCB1. This resulted in the synthesis of a shortened SMARCB1 protein due to reinitiation of translation at an AUG-codon downstream of the nonsense variant [56]. When analyzing samples one should also keep in mind the possible presence of cells containing unaffected DNA in the sense of a parental germline mosaicism, as it is typical for familiar schwannomatosis [57]. Gonadal mosaicism of the parents appears to be regularly involved in hereditary RT [10, 47, 58-60].

It further appears useful not only to involve location and specific type of a variant, but also its time point during development and to further have a look at additional genes that may be required for tumorigenesis. Information about temporal distribution of variants is only given in an experimental setting. According to Han et al., tamoxifenmediated inactivation of SMARCBI in mice results in different phenotypes depending on time of injection. Mice develop RT, when SMARCB1 is inactivated in a short timeframe between E6 and E10, while injection at earlier and later stages of development leads to embryonic lethality, hepatotoxicity, or T-cell lymphoma [61], suggesting a cellular period of vulnerability to truncating variants that result in RT. Human variant carriers not receiving a second hit in the critical timeframe might accumulate additional variants later in life, which is in line with the 3-step/4-hit model of pathogenesis for schwannoma. Here, an adjacent gene, NF2, contributes to tumorigenesis: Initially, a constitutional variant inactivates one copy of SMARCB1, followed by a loss of its second copy together with the adjacent NF2 and an inactivating variant of the remaining wild-type $N F 2$ as somatic events [62]. Comparable mechanisms of pathogenesis have been assumed for meningioma [50]. These considerations match well the clinical data: Patients suffering from schwannoma and meningioma fall ill significantly later in life, as do carriers of non-truncating SMARCBI variants. This also holds true for the three families suffering from schwannoma as well as RT. Interestingly, the effect may also be observed within a group of patients suffering from low-grade SMARCB1mutated tumors. Comparing variant carriers with or without truncating variants, the median age of tumor onset is considerably lower for the former (24.5 vs. 39.5 years), possibly indicating a residual protein activity in the case of a missense variant. Individuals, who are diagnosed with an $\mathrm{RT}$ in adolescence or adulthood, are exceedingly rare. One may thus hypothesize the persistence of stem cells that have retained their vulnerability for a critical second hit. Indeed, RT cells exhibit common features of embryonic stem cells [63], although the cell of origin of RT yet remains to be elucidated.

For investigating possible pathomechanisms, asymptomatic carriers of SMARCBI germline variants are of particular interest. All unaffected carriers involved in our study are related to diseased persons. Of note, only oncogenic variants appear in healthy variant carriers, and we do not know of the latter kindred with CSS or NCBRS patients. The oncogenic variants do not show any preference of gender, age, or degree of kinship, confirming an accidental second hit that might hit or not hit an individual as the underlying cause for disease pathogenesis. Interestingly, all types of variants except in-frame variants or deletions larger than two exons are represented in this group. This is in sharp contrast to the large number of RT patients with extended SMARCB1 deletions (38\%) and might indicate full penetrance for this kind of variant, whereas singlenucleotide variants are characterized by incomplete penetrance as well as variable expression. Nevertheless, one should keep in mind the possibility of parental germ cell mosaicism in these cases [60], which is why relatives should be screened thoroughly for such events. Remarkably, the amount of unaffected variant carriers differs among genes and it is much more likely to find a patient with healthy, variant-bearing relatives in families affected by SMARCA4 (23\% of all SMARCA4 variant carriers reviewed in this article) or SMARCE1 variants $(25 \%)$ than it is in those with SMARCB1 driven disease (5\%). This in line with the assumption that the 22q.11.2 locus is a highly instable region susceptible to variants, deletions and translocations, and numerous diseases emerging from genomic alterations at this site reflect this observation (DiGeorgesyndrome, OMIM \# 188400; Shprintzen-syndrome, OMIM 
\# 192430; 22q11.2 duplication syndrome, OMIM \# 608363; Emanuel-syndrome, OMIM \# 609029; Supernumerary der(22)t(8;22)-syndrome, OMIM \# 613700; CSS3, OMIM \# 614608; RTPS1, OMIM \# 609322; SWNTS1, OMIM \# 162091).

Certain entities can be attributed to germline variants in specific genes. Having a closer look on their distribution, it becomes clear that for every single gene other principles for tumorigenesis may be valid and that they differ from what is known about somatic alterations. Somatic variants in $A R I D 1 / 2$ genes are widely known to occur in a broad variety of cancers [64]. In contrast, truncating germline variants in ARIDIA, ARIDIB, or ARID2 predispose to CSS [22, 34, 35, 65] and hardly ever lead to tumor formation. On the other hand, both, truncating and non-truncating germline variants in $S M A R C B 1[10,51]$, regularly result in tumor development. Of note, the latter can also be associated with a CSS phenotype without tumor predisposition, when the $3^{\prime}$ end of the gene is affected [22, 34, 35]. Variants in SMARCE1 seem to be restricted to generation of meningioma or CSS [38], whereas SCCOHT only appears in the context of SMARCA4 variants [20]. Heterozygous missense variants involving SMARCA4 regularly lead to CSS [34, 35], indicating for a dominant negative effect for this type of germline alteration. Only one case of RT emerging from SMARCA4 missense variant is known so far [12]. Regarding SMARCB1 and SMARCA4, malignancy usually seems to be associated with loss of protein function due to truncating variants or complete deletions; however, the question arises how far splice-site and, rarely, missense variants detected in malignant tumors may exert a functional impact.

In the light of these obvious and largely regular patterns of disease expression one must assume substantial functional differences for the core units the complex is composed of. The BAF complex is expressed ubiquitously in all cells, with changes in subunit composition depending on the tissue. Its core units, although expressed consistently, may also exhibit different levels of activity or manners of function in their role as a chromatin remodeler, depending on cell type, stage of cell development and outside influences, finally cellular necessities of transcriptional action. This could explain differing impact of comparable variants at least in parts. Recent findings have underlined the importance of the BAF complex in terms of lineage specification and cell differentiation, and for particular subunits functional effects can be hypothesized. SMARCB1 seems to play a crucial role for the functional integrity of the whole complex, as its loss leads to reduced binding to promoters and enhancers, thereby pertubating cell differentiation, while its level at super-enhancers and, of note, cell survival is barely affected [7]. However, further investigation with focus on the relevance and function of specific $B A F$ subunits is needed to get a deeper understanding of how $B A F$-related diseases emerge and, finally, can be cured.

In conclusion, we have reviewed a large series of $B A F$ germline variant carriers, matching clinical with genetic data, and discussing possible mechanisms of pathogenesis. We have gathered information of 577 patients of over 400 families and include 43 individuals that have not been published so far.

Our data highlight the impact of truncating germline variants and their influence on disease development. We provide a broad overview of what is known so far and give useful impulses for further investigation in terms of understanding the complex $B A F$ genetics.

Acknowledgements US and RS are supported by the Fördergemeinschaft Kinderkrebs-Zentrum Hamburg, US in addition by the Deutsche Krebshilfe. MH is supported by DFG (HA 3060/5-1) and IZKF Münster (Ha3/019/15). RSi is supported by the KinderKrebsInitiative Buchholz/Holm-Seppensen.

\section{Compliance with ethical standards}

Conflict of interest The authors declare that they have no conflict of interest.

\section{References}

1. Nguyen H, Sokpor G, Pham L, et al. Epigenetic regulation by BAF (mSWI/SNF) chromatin remodeling complexes is indispensable for embryonic development. Cell Cycle. 2016;15:1317-24.

2. Wu Q, Madany P, Dobson JR, et al. The BRG1 chromatin remodeling enzyme links cancer cell metabolism and proliferation. Oncotarget. 2016;7:38270-81.

3. Jubierre L, Soriano A, Planells-Ferrer L, et al. BRG1/SMARCA4 is essential for neuroblastoma cell viability through modulation of cell death and survival pathways. Oncogene. 2016;35:5179-90.

4. Narlikar GJ, Sundaramoorthy R, Owen-Hughes T. Mechanisms and functions of ATP-dependent chromatin-remodeling enzymes. Cell. 2013;154:490-503.

5. Biegel JA, Zhou JY, Rorke LB, Stenstrom C, Wainwright LM, Fogelgren B. Germ-line and acquired mutations of INI1 in atypical teratoid and rhabdoid tumors. Cancer Res. 1999; 59:74-79.

6. Alver $\mathrm{BH}, \mathrm{Kim} \mathrm{KH}, \mathrm{Lu} \mathrm{P}$, et al. The SWI/SNF chromatin remodelling complex is required for maintenance of lineage specific enhancers. Nat Commun. 2017;8:14648.

7. Wang X, Lee RS, Alver BH, et al. SMARCB1-mediated SWI/ SNF complex function is essential for enhancer regulation. Nat Genet. 2017;49:289-95

8. Kadoch C, Crabtree GR. Mammalian SWI/SNF chromatin remodeling complexes and cancer: mechanistic insights gained from human genomics. Sci Adv. 2015;1:e1500447.

9. Kadoch C, Hargreaves DC, Hodges C, et al. Proteomic and bioinformatic analysis of mammalian SWI/SNF complexes identifies extensive roles in human malignancy. Nat Genet. 2013;45:592-601.

10. Bourdeaut F, Lequin D, Brugieres L, et al. Frequent hSNF5/INI1 germline mutations in patients with rhabdoid tumor. Clin Cancer Res. 2011;17:31-38. 
11. Schneppenheim R, Fruhwald MC, Gesk S, et al. Germline nonsense mutation and somatic inactivation of SMARCA4/BRG1 in a family with rhabdoid tumor predisposition syndrome. Am J Hum Genet. 2010;86:279-84.

12. Hasselblatt M, Nagel I, Oyen F, et al. SMARCA4-mutated atypical teratoid/rhabdoid tumors are associated with inherited germline alterations and poor prognosis. Acta Neuropathol. 2014;128:453-6.

13. Biegel JA, Busse TM, Weissman BE. SWI/SNF chromatin remodeling complexes and cancer. Am J Hum Genet. 2014;166C:350-66.

14. Johann PD, Hovestadt V, Thomas $\mathrm{C}$ et al. Cribriform neuroepithelial tumor: Molecular characterization of a SMARCB1deficient non-rhabdoid tumor with favorable long-term outcome. Brain Pathol. 2017;27:411-18

15. Carter JM, O'Hara C, Dundas G, et al. Epithelioid malignant peripheral nerve sheath tumor arising in a schwannoma, in a patient with "neuroblastoma-like" schwannomatosis and a novel germline SMARCB1 mutation. Am J Surg Pathol. 2012;36:154-60.

16. Smith MJ, Wallace AJ, Bowers NL, Eaton H, Evans DGR. SMARCB1 mutations in schwannomatosis and genotype correlations with rhabdoid tumors. Cancer Genet. 2014;207:373-8.

17. Le Loarer F, Zhang L, Fletcher CD, et al. Consistent SMARCB1 homozygous deletions in epithelioid sarcoma and in a subset of myoepithelial carcinomas can be reliably detected by FISH in archival material. Gene Chromosom Cancer. 2014;53:475-86.

18. Paganini I, Sestini R, Cacciatore M, et al. Broadening the spectrum of SMARCB1-associated malignant tumors: a case of uterine leiomyosarcoma in a patient with schwannomatosis. Hum Pathol. 2015;46:1226-31.

19. Witkowski L, Carrot-Zhang J, Albrecht S, et al. Germline and somatic SMARCA4 mutations characterize small cell carcinoma of the ovary, hypercalcemic type. Nat Genet. 2014;46:438-43.

20. Witkowski L, Goudie C, Foulkes WD, McCluggage WG. Smallcell carcinoma of the ovary of hypercalcemic type (malignant rhabdoid tumor of the ovary): a review with recent developments on pathogenesis. Surg Pathol Clin. 2016;9:215-26.

21. Smith MJ. Germline and somatic mutations in meningiomas. Cancer Genet. 2015;208:107-14.

22. Wieczorek D, Bogershausen N, Beleggia F, et al. A comprehensive molecular study on Coffin-Siris and Nicolaides-Baraitser syndromes identifies a broad molecular and clinical spectrum converging on altered chromatin remodeling. Hum Mol Genet. 2013;22:5121-35.

23. Schrier Vergano S, Santen G, Wieczorek D, Wollnik B, Matsumoto N, Deardorff MA. Coffin-Siris syndrome. In: Pagon RA, Adam MP, Ardinger HH, Wallace SE, Amemiya A, Bean LJH, Bird TD, Fong C-T, Mefford HC, Smith RJH, Stephens K, editors. GeneReviews(R). Seattle: University of Washington; 1993.

24. Sousa SB, Hennekam RC. Phenotype and genotype in NicolaidesBaraitser syndrome. Am J Med Genet. 2014;166C:302-14.

25. Gossai N, Biegel JA, Messiaen L, Berry SA, Moertel CL. Report of a patient with a constitutional missense mutation in SMARCB1, Coffin-Siris phenotype, and schwannomatosis. Am J Med Genet. 2015;167A:3186-91.

26. Tsurusaki Y, Okamoto N, Ohashi H. Mutations affecting components of the SWI/SNF complex cause Coffin-Siris syndrome. Nat Genet. 2012;44:376-8.

27. Vengoechea J, Carpenter L, Zarate YA. Papillary thyroid cancer in a patient with interstitial 6q25 deletion including ARID1B. Am J Med Genet. 2014;164A:1857-9.

28. Sredni ST, Tomita T. Rhabdoid tumor predisposition syndrome. Pediatr Dev Pathol. 2015;18:49-58.

29. Taylor MD, Gokgoz N, Andrulis IL, Mainprize TG, Drake JM, Rutka JT. Familial posterior fossa brain tumors of infancy secondary to germline mutation of the hSNF5 gene. Am J Hum Genet. 2000;66:1403-6.

30. Sevenet N, Lellouch-Tubiana A, Schofield D, et al. Spectrum of hSNF5/INI1 somatic mutations in human cancer and genotypephenotype correlations. Hum Mol Genet. 1999;8:2359-68.

31. Forest F, David A, Arrufat S, et al. Conventional chondrosarcoma in a survivor of rhabdoid tumor: enlarging the spectrum of tumors associated with SMARCB1 germline mutations. Am J Surg Pathol. 2012;36:1892-6.

32. Hulsebos TJM, Kenter S, Siebers-Renelt U, Hans V, Wesseling P, Flucke U. SMARCB1 involvement in the development of leiomyoma in a patient with schwannomatosis. Am J Surg Pathol. 2014;38:421-5.

33. Ammerlaan ACJ, Ararou A, Houben MPWA, et al. Long-term survival and transmission of INI1-mutation via nonpenetrant males in a family with rhabdoid tumour predisposition syndrome. Br J Cancer. 2008;98:474-9.

34. Tsurusaki Y, Okamoto N, Ohashi H, et al. Coffin-Siris syndrome is a SWI/SNF complex disorder. Clin Genet. 2014;85:548-54.

35. Santen GWE, Aten E, Vulto-van Silfhout AT, et al. Coffin-Siris syndrome and the BAF complex: genotype-phenotype study in 63 patients. Hum Mutat. 2013;34:1519-28.

36. Abdul-Rahman O. Nicolaides-Baraitser syndrome. In: Pagon RA, Adam MP, Ardinger HH, Wallace SE, Amemiya A, Bean LJH, Bird TD, Fong C-T, Mefford HC, Smith RJH, Stephens K, editors. GeneReviews(R). Seattle: University of Washington; 1993.

37. Smith MJ, O'Sullivan J, Bhaskar SS, et al. Loss-of-function mutations in SMARCE1 cause an inherited disorder of multiple spinal meningiomas. Nat Genet. 2013;45:295-8.

38. Smith MJ, Wallace AJ, Bennett C, et al. Germline SMARCE1 mutations predispose to both spinal and cranial clear cell meningiomas. J Pathol. 2014;234:436-40.

39. Gerkes EH, Fock JM, den Dunnen WFA, et al. A heritable form of SMARCE1-related meningiomas with important implications for follow-up and family screening. Neurogenetics. 2016;17:83-89.

40. Wu JN, Roberts CWM. ARID1A mutations in cancer: another epigenetic tumor suppressor? Cancer Discov. 2013;3:35-43.

41. Sausen M, Leary RJ, Jones S, et al. Integrated genomic analyses identify ARID1A and ARID1B alterations in the childhood cancer neuroblastoma. Nat Genet. 2013;45:12-17.

42. Stephens PJ, Tarpey PS, Davies H, et al. The landscape of cancer genes and mutational processes in breast cancer. Nature. 2012;486:400-4.

43. Kleefstra T, Kramer JM, Neveling K, et al. Disruption of an EHMT1-associated chromatin-modification module causes intellectual disability. Am J Hum Genet. 2012;91:73-82.

44. Kehrer-Sawatzki H, Farschtschi S, Mautner V-F, Cooper DN. The molecular pathogenesis of schwannomatosis, a paradigm for the co-involvement of multiple tumour suppressor genes in tumorigenesis. Hum Genet. 2017;136:129-48.

45. Swensen JJ, Keyser J, Coffin CM, Biegel JA, Viskochil DH, Williams MS. Familial occurrence of schwannomas and malignant rhabdoid tumour associated with a duplication in SMARCB1. J Med Genet. 2009;46:68-72.

46. Bosse KR, Shukla AR, Pawel B, et al. Malignant rhabdoid tumor of the bladder and ganglioglioma in a 14 year-old male with a germline 22q11.2 deletion. Cancer Genet. 2014;207:415-9.

47. Bruggers CS, Bleyl SB, Pysher T, et al. Clinicopathologic comparison of familial versus sporadic atypical teratoid/rhabdoid tumors $(\mathrm{AT} / \mathrm{RT})$ of the central nervous system. Pediatr Blood Cancer. 2011;56:1026-31.

48. Kordes U, Bartelheim K, Modena P, et al. Favorable outcome of patients affected by rhabdoid tumors due to rhabdoid tumor predisposition syndrome (RTPS). Pediatr Blood Cancer. 2014;61:919-21. 
49. Janson K, Nedzi LA, David O, et al. Predisposition to atypical teratoid/rhabdoid tumor due to an inherited INI1 mutation. Pediatr Blood Cancer. 2006;47:279-84.

50. van den Munckhof P, Christiaans I, Kenter SB, Baas F, Hulsebos TJM. Germline SMARCB1 mutation predisposes to multiple meningiomas and schwannomas with preferential location of cranial meningiomas at the falx cerebri. Neurogenetics. 2012;13:1-7.

51. Boyd C, Smith MJ, Kluwe L, Balogh A, Maccollin M, Plotkin SR. Alterations in the SMARCB1 (INI1) tumor suppressor gene in familial schwannomatosis. Clin Genet. 2008;74:358-66.

52. Moes-Sosnowska J, Szafron L, Nowakowska D et al. Germline SMARCA4 mutations in patients with ovarian small cell carcinoma of hypercalcemic type. Orphanet J Rare Dis. 2015;10:32.

53. Witkowski L, Donini N, Byler-Dann R, et al. The hereditary nature of small cell carcinoma of the ovary, hypercalcemic type: two new familial cases. Fam Cancer. 2017;16:395-99.

54. Versteege I, Sevenet N, Lange J, et al. Truncating mutations of hSNF5/INI1 in aggressive paediatric cancer. Nature. 1998;394:203-6.

55. Santen GWE, Kriek M, van Attikum H. SWI/SNF complex in disorder: SWItching from malignancies to intellectual disability. Epigenetics. 2012;7:1219-24.

56. Hulsebos TJM, Kenter S, Verhagen WIM, Baas F, Flucke U, Wesseling P. Premature termination of SMARCB1 translation may be followed by reinitiation in schwannomatosis-associated schwannomas, but results in absence of SMARCB1 expression in rhabdoid tumors. Acta Neuropathol. 2014;128:439-48.

57. Patil S, Perry A, Maccollin M, Dong S, Betensky RA, Yeh T-H, et al. Immunohistochemical analysis supports a role for INI1/
SMARCB1 in hereditary forms of schwannomas, but not in solitary, sporadic schwannomas. Brain Pathol. 2008;18:517-19.

58. Eaton KW, Tooke LS, Wainwright LM, Judkins AR, Biegel JA. Spectrum of SMARCB1/INI1 mutations in familial and sporadic rhabdoid tumors. Pediatr Blood Cancer. 2011;56:7-15.

59. Sevenet N, Sheridan E, Amram D, Schneider P, Handgretinger R, Delattre O. Constitutional mutations of the hSNF5/INI1 gene predispose to a variety of cancers. Am $\mathrm{J}$ Hum Genet. 1999;65:1342-8.

60. Gigante L, Paganini I, Frontali M, Ciabattoni S, Sangiuolo FC, Papi L. Rhabdoid tumor predisposition syndrome caused by SMARCB1 constitutional deletion: prenatal detection of new case of recurrence in siblings due to gonadal mosaicism. Fam Cancer. 2016;15:123-6.

61. Han Z-Y, Richer W, Freneaux P, et al. The occurrence of intracranial rhabdoid tumours in mice depends on temporal control of Smarcb1 inactivation. Nat Commun. 2016;7:10421.

62. Plotkin SR, Blakeley JO, Evans DG, et al. From genetics to diagnostic criteria. Am J Med Genet A. 2013;161A:405-16.

63. Deisch J, Raisanen J, Rakheja D. Immunohistochemical expression of embryonic stem cell markers in malignant rhabdoid tumors. Pediatr Dev Pathol. 2011;14:353-9.

64. Lin C, Song W, Bi X, et al. Recent advances in the ARID family: focusing on roles in human cancer. OncoTargets Ther. 2014;7:315-24.

65. Bramswig NC, Caluseriu O, Ludecke H-J, et al. Heterozygosity for ARID2 loss-of-function mutations in individuals with a Coffin-Siris syndrome-like phenotype. Hum Genet. 2017;136:297-305.

\section{Affiliations}

\section{Till Holsten ${ }^{1,2,3} \cdot$ Susanne Bens ${ }^{4} \cdot$ Florian Oyen $^{1} \cdot$ Karolina Nemes $^{5} \cdot$ Martin Hasselblatt $^{6} \cdot$ Uwe Kordes $^{1} \cdot$ Reiner Siebert ${ }^{4}$ - Michael C. Frühwald ${ }^{5}$ - Reinhard Schneppenheim ${ }^{1}$ • Ulrich Schüller (iD) $1,2,3$}

1 Department of Pediatric Hematology and Oncology, University Medical Center Hamburg-Eppendorf, Hamburg, Germany

2 Institute of Neuropathology, University Medical Center HamburgEppendorf, Hamburg, Germany

3 Research Institute Children's Cancer Center Hamburg, Hamburg, Germany
4 Institute of Human Genetics, University of Ulm \& Ulm University Hospital, Ulm, Germany

5 Children's Hospital Augsburg, Swabian Children's Cancer Center, Augsburg, Germany

6 Institute of Neuropathology, University Hospital Münster, Münster, Germany 